\title{
Some Challenges in the Design and Education OF INTELLIGENT MACHINES AND SYSTEMS
}

\author{
Witold Kinsner and Yingxu Wang* \\ Department of Electrical and Computer Engineering \\ University of Manitoba, Winnipeg, MB, Canada R3T 5V6 \\ and *Department of Electrical and Computer Engineering \\ University of Calgary, Calgary AB Canada T2N 1N4 \\ w.kinsner@ieee.org
}

\begin{abstract}
Numerous attempts are being made to develop machines that could act not only autonomously, but also in an increasingly intelligent and cognitive manner. Such cognitive machines ought to be aware of their environments which include not only other machines, but also human beings. Such machines ought to understand the meaning of information in more human-like ways by grounding knowledge in the physical world and in the machines' own goals. The motivation for developing such machines range from self-evidenced practical reasons such as the expense of computer maintenance, to wearable computing in health care, and gaining a better understanding of the cognitive capabilities of the human brain.

To achieve such an ambitious goal requires solutions to many problems, ranging from human perception, attention, concept creation, cognition, consciousness, executive processes guided by emotions and value, and symbiotic conversational human-machine interactions.

This paper discusses some of the challenges emerging from this new design paradigm, including systemic problems, design issues, teaching the subjects to undergraduate students in electrical and computer engineering programs, research related to design.
\end{abstract}

Keywords: Design paradigms, adaptive machines, intelligent machines, cognitive machines; challenges in design and education.

\section{INTRODUCTION}

Engineers cause change through their knowledge, skills and professional activities [Koen03; p.11], [EdHo08]. One of the major roles of engineers is to design, build and test new physical or logical systems that have not existed before. A system is a deliberate arrangement of parts (such as hardware and software components, functional units, subsystems, procedures, or people and facilities) required to achieve a desired goal and specific objectives (e.g., [HoWo05]). Following the scientific method, with its physical and mathematical principles augmented by ergonomics and aesthetics, a system is first specified, then simulated and emulated if necessary, and its different parts (subsystems) are built and put together, tested and installed in an environment for which it was intended. Finally, field testing and operational observations provide feedback for improvements and modifications of the system.

If the system is simple (i.e., linear, with few static noninteracting components), many well-established design techniques can be used. If the system is complex (nonlinear, with interacting components), the design techniques must be much more involved including many heuristics. When the system must involve human operators (not users), even more complex design techniques must be considered. This paper describes some of the design challenges brought about by the emerging dynamical systems, as well as intelligent and cognitive machines and systems [Kins07], [Kins09]. These developments require profound changes to the design process and education as dictated by the new scientific and engineering principles involved. Some of these issues are being tackled by cognitive informatics [Wang06].

\section{ENGINEERING DESIGN}

\subsection{An Engineer}

The American College Dictionary and other dictionaries define an engineer as a person who is not only versed in the design, construction and use of machines, but also is capable of employing the innovative and methodological application of scientific knowledge and technology to produce a device, or a system, or a process, all intended to satisfy human needs, subject to technological, economic and environmental constraints.

Similar definitions are provided by the engineering accreditation bodies such as the Canadian Engineering Accreditation Board (CEAB) of Engineers Canada (formerly the Canadian Council of Professional Engineers, CCEP) [CEAB06], and the ABET Engineering Accreditation Commission (formerly the Accreditation Board of Engineering and Technology) in the USA [ABET06].

The definitions stress design and implementation through innovative and methodological application of knowledge (not just of information, or even worse, data). As we shall see, innovation and creativity are of particular importance to the design of intelligent systems. We shall also see that the design process of such systems requires a well synchronized team of engineers and other professional from non-engineering disciplines, rather than an ensemble of 
isolated individuals. In fact, a concept of the world-class engineer has been developed at academics and industry [Leon95].

\subsection{The Engineering Design Process (EDP)}

What is an engineering design process (EDP)? The process of developing a product is based on a design philosophy [Koen03] and involves a number of steps that guide the designer from the concept to the implementation and testing in the field. All the engineering accreditation bodies define engineering design clearly. For example, according to the Canadian CEAB [CEAB06; p. 12], engineering design integrates mathematics, basic sciences, engineering sciences and complementary studies in developing elements, systems and processes to meet specific needs. It is a creative, interactive and often open-ended process subject to constraints which may be governed by standards or legislation to varying degrees, depending upon the discipline. These constraints may relate to economic, health, safety, environmental, social, or other pertinent interdisciplinary factors.

According the American ABET [ABET06], engineering design is the process of devising a system, component or process to meet desired needs. It is a decision-making process (often interactive), in which the basic sciences, mathematics, and engineering sciences are applied to convert resources optimally to meet a stated objective. Among the fundamental elements of the design process are the establishment of objectives and criteria, synthesis, analysis, construction, testing and evaluation.

The International Technology Education Association (ITEA) defines [ITEA07] engineering design ad the systematic and creative application of scientific and mathematical principles to practical ends such as the design, manufacture and operation of efficient and economic structure, machines, processes, and systems.

Although the definitions differ in several marked aspects, they point to some common elements of the design process, including specifications, analysis, synthesis, implementation and testing, regardless of the discipline. The actual design methodologies may also differ considerably, depending on the discipline (e.g., computer engineering) and area such as integrated circuit design, embedded systems, and software systems, as described in the next section.

\subsection{Two Types of the Engineering Design Process}

Although design involves fairly universal ideas [Broo75] [Broo10], is there a single approach to the engineering design process? Cross [Cros00] distinguishes two types of processes: prescriptive (algorithmic) and descriptive (open-ended, holistic, gestalt). The prescriptive (procedural) approach involves the basic phases identified in the previous section. It can be applied to very simple, well established designs in which several conditions are satisfied: (i) the problem and needs are well understood, (ii) the requirements are known and can be improved from previous similar cases, and (iii) the implementation and product introduction have low risk. Although feedback (i.e., revisiting previous phases) occurs in the process to correct or improve product performance, the process is sequential.

More complex machines and systems require the descriptive approach in which various phases of the design process do not follow sequentially, but are interconnected in the sense that their future impact must be considered at each other phase at all times, as shown in Fig. 1.

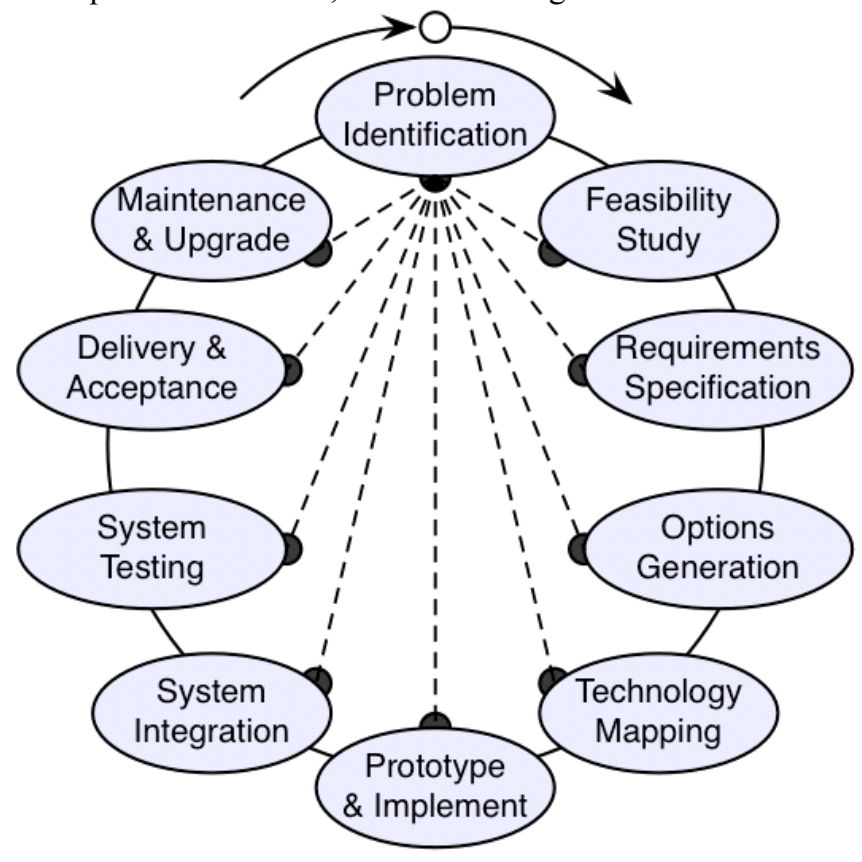

Fig. 1 A descriptive approach to the engineering design process. (After [FoCo05, p. 4])

The process usually starts from the problem identification phase in which the need for the product (i.e., a device, or a module, or a system) is established clearly. Mistakes are frequent in this phase due to either misjudgment of the current needs or unexpected changes in the future economic or environmental conditions. Such mistakes may be very costly. To minimize this risk related to known factors, various procedures have been developed. The dashed lines emanating from this phase to all the other nine phases indicate an awareness of the limitations and constraints of the other stages in the design process. Although they do not imply any actual visitations of the other phases at this stage of the process, they indicate the parallel nature of this model. The simultaneous awareness increases with the experience of thee design team, and could improve the accuracy of the problem formulation. If similar lines were drawn from each phase, a fully connected graph would result (see [FoCo05, p. 4]). This is not done in Fig. 1 to avoid overcrowding.

A preliminary design team is often established at this 
stage to conduct a feasibility study in order to identify the scientific and engineering principles involved, as well as the available technologies and prior art (available solutions). This establishes if the product is feasible within the available resources. Notice again that although not shown by the dashed lines in Fig 1, this stage is also connected to all the other nine phases, with the strongest link going to the previous phase.

During the requirements specification phase, the team describes what the product is, and what it must do in order to satisfy the needs. The specifications must be formulated in writing as both text and graphics in the form of a functional (architectural) block diagram. The block diagram represents a partitioning of the system into subsystems (modules), and identifies all the interfaces between the various functional units, together with their levels of difficulty. This requirements stage is often assisted by computer-based tools and languages in order to improve and maintain product documentation. Although this phase does not provide an optimal solution, it is a guide for the entire remaining design process.

The distinguishing feature of the descriptive (openended) design approach is demonstrated in the next option generation phase. By developing the necessary solution to the problem, a cost function must also be established to perform a constrained optimization and grade the solutions. This phase is often marked by extreme innovation and creativity.

The technology mapping phase converts the selected optimal functional (architectural) solution into a detailed technological solution. This transformed product organization involves all the specific available parts, their specific interconnections (buses), and specific protocols to communicate between the components. Based on the technology mapping, the system partitioning may also be revisited to search for a better solution. To minimize logical mistakes, this stage often uses computer-based simulators (software), or emulators (software augmented with some actual hardware). Logical verification and testing are integral part of this phase. Verification is intended to prove that each module does what it should, while testing establishes its performance limits. Testing may be very time consuming at this stage due to the complexity of test vectors.

The prototyping and implementation phase provides the proof-of-concept because specific components are acquired, assembled and tested as physical prototypes in order to gain understanding of the physical limitations of the actual implementation. Prior experience with similar components may not be sufficient because of the technological changes in their manufacture. The subsystems are often implemented using rapid prototyping such as the field-programmable gate arrays (FPGAs) from Xilinx (e.g., [Coff00]), or other programmable logic devices (PLDs) such as the complex PLDs (CPLDs) from Altera (e.g., [Duec01]), or 3D printing and stereolithography [Castl07]. The prototypes further remove either logical or physical mistakes such as speed limitation. This phase also includes verification and testing.

The system integration phase is responsible for bringing all the subsystems together. This phase is often very difficult because the functional subsystems may not work together due to interface problems such as timing in the protocols, or improper utilization of resources such as memory conflicts. Real-time systems are particularly difficult to integrate.

The system verification and testing phase is an extension of similar activities in the technology mapping and prototyping phases. The system is verified against standards, and the overall performance is tested so that it is consistent with the system specifications.

The last two stages are delivery and acceptance and maintenance and upgrading. They follow procedures agreed upon in the preliminary phases of the product design process. The last stage may be very costly if the usable product lifetime is long. Since, for some systems such as computer installations, the cost of maintenance may be up to ten times the cost of the equipment, the systems must be designed in new ways. For example, autonomic computing [Kins05], [Kins07] and intelligent systems may alleviate some of the problems.

Notice that although the ten phases apply to any engineering design process, their description has been slanted towards computer engineering to reflect the experience of these authors.

\section{AREA-SPECIFIC EDP}

The general EDP is often translated into an area-specific EDP. For example the electronic circuit EDP has evolved from discrete components, to integrated circuits, and to fieldprogrammable gate arrays (FPGAs). Each variation on the technology resulted in major modifications of the EDP over the years.

Development of software, and particularly software for real-time systems, is also a complex undertaking, and requires a dedicated software EDP.

One of the important activities in computer engineering is the design of embedded systems. Embedded systems are hardware-software computing and controlling systems encapsulated into larger electromechanical systems to perform dedicated operations in a way that is often inconspicuous to the outside observer. This embedded systems EDP, together with the electronic circuit and software EDP, is described in [Kins09].

\section{EVOLUTION OF SYSTEMS}

Rapid shifts are being observed in technological and computing paradigms, as described in [Kins07] and [Kins09]. These remarkable shifts have been necessitated by the system complexity, which now exceeds our ability to maintain them [GaCo03], while being facilitated by new developments in technology, intelligent signal processing, 
and machine learning [HPSM06]. By necessity, the new systems are becoming cognitive. In general, the intent of such cognitive systems is to improve their performance in changing environments, to reduce waste in resource utilization, to help human operators interact with the controlled process, to guard against catastrophic failure, to age gracefully, and to provide a test-bed for learning about cognition and cognitive processes. Examples include those proposed by Haikonen [Haik03], Franklin [Fran95], Aleksander [Alek98], Taylor [Tayl03], with specific examples such as cognitive radio [Hayk05], cognitive radar [Hayk06], active audition [HaCh05], and cognitive robots, affective computing, and autonomic computing. Without this framework, the challenges of designing intelligent and cognitive systems might not be appreciated fully.

\section{CHALLENGES IN THE DESIGN OF COMPLEX SYSTEMS}

The previous sections provided a review of the commonalities and differences in the engineering design process (EDP) of electronic hardware, software and embedded systems. These models are well understood, and are used in practice in industry, as well as in research and development $(R \& D)$ organizations. When a limitation of a specific model is identified, the model is improved. This has been practised for decades. So, is there a problem? Yes, there may be a fundamental problem with all the existing EDP models if the complexity of a system increases beyond what is known (i.e., the system becomes unmanageable), or when limited resources prevent the use of the system, or when the system includes human operators as an integral part of the system. In such cases, intelligent and cognitive systems ought to be considered. However, there is no EDP model developed for such systems. This discussion may be helpful in the development of such a model. The discussion is grouped into several topics related to outsourcing of manufacturing, the new role of design teams, complex systems, the EDP, teaching, and research.

\subsection{Outsourcing of Manufacturing}

One of the major challenges in EDP is the outsourcing of manufacturing in our global economy. How can we sustain design without knowing how to manufacture the designs? For example, in chip manufacturing, since the physical parameters of the wafer may change from day to day, they are measured and fed back into the parameters used in simulation of the circuit to estimate its speed and other performance criteria. Without knowing the details of manufacturing, our designs cannot be optimal, or even may fail prematurely.

The problem is compounded by the number of engineers graduated in those countries that are handed over the manufacturing. For example in 2006, the number of graduating engineers was around 600,000 in China and around half of that in India, while North America graduated just around one tenth of that number. One of the outcomes of not meeting this challenge is the declining enrollment in some areas of engineering, as the perception is that there will be no engineering jobs here.

\subsection{The Role of Engineers and Engineering Teams}

The engineer must be not only a good designer, but also a user's advocate (user-centred designs). The engineer must also be a design diplomat, capable of guiding the design in a team, enhancing the environment of all the team members by balancing their interests, and keeping the client fully involved and aware of the status of the project.

The complexity of intelligent systems requires teams of professionals whose individual detailed knowledge of the entire system may be next to impossible. Many teams may also be distributed physically in a city, country, or even the world. In the later case, the teams may not be working at the same time due to the opposite time zones. Encapsulation of expertise and reusable software may be necessary in such cases.

\subsection{System Challenges}

Autonomic Computing: Designing intelligent and cognitive systems is a long-term goal. Autonomic computing (AC) is a scaled-down form of cognitive machines [GaCo03], [IBM06], [Kins05], [Kins07], [Wang03], [Wang04], [Wang06]. The systems are evolving earnestly because the cost-performance of hardware improvements (speed and capacity) have lead to escalating complexity of software (features and interfaces). However, this increased complexity requires elaborate managing systems that are now six to ten times the cost of the equipment itself. Autonomic computing is intended to simplify this problem by making the systems self-configuring, self-optimizing, self-organizing, self-healing, self-protecting, and selftelecommunicating, thus leading to increased reliability, robustness, and dynamic flexibility. This involves not only the traditional fault tolerant computing (i.e., tolerating hardware and software faults), but also tolerating various faults made by human operators and users, thus shifting attention from the mean-time-between failures (MTBF) to the mean-time-to-recover (MTTR) in order to make the systems more available. AC applies to both desktop computing, portable computing, pervasive computing, and embedded systems.

A number of new concepts must also be developed, such as the real-time process algebra (RTPA) [Wang02]. RTPA can provide a multidimensional (space, time and action) representation of the behaviour of autonomous systems.

Mechatronics: Many of the developments of intelligent machines involve mechatronics (e.g., [Bolt03], [Ceti07]) which is a system-level approach to designing electromechanical systems. It combines several engineering 
disciplines, including mechanical, electrical, electronic, control, wireless, and computer, with an extended embedded systems design methodology. Machines that perform one task are no longer in great demand. Flexible machines require control, human-machine interfaces (HMI), networking, remote machine monitoring and reporting over the Internet. However, such complexities increase cost, risk, and design time. Mechatronics-based approach mitigates some of the problems.

Networking: How can we provide resilient networking for intelligent and cognitive machines? Should the networking be wired or wireless? How can the Internet be used for telerobotics and telepresence in view of the unpredictable delays on the network? Boosting the Internet routers will not solve the problem. Reconfigurable hardware and networkembedded computational resources will not solve the problem either. Can the network be redesigned to make the attached computing resources not only a distributed computing system, but also a cognitive global computer?

\subsection{Direct Challenges Within the EDP}

The EDP itself has many challenges, particularly when applied to complex systems, including rapid EDP cycle, requirements, specifications, design and evaluation, hardware and sensors, and software, as discussed next.

Short EDP Cycle: The turn-around time for product specification, design and delivery has been decreasing not only in embedded systems and mechatronics, but also in automotive and aerospace industries with very complex environments spanning many departments and partners. Rapid evaluation and alternative designs require massive computing resources. Can grid computing be helpful to resolve this problem? Grid computing is a service-oriented architecture approach that uses open standards and resource virtualization to make distributed computing possible over the Internet. An example may be IBM's Grid-Aided Engineering (GAE) [IBM07].

Requirements: Identifying the current needs for a product, its characteristics, functionality, reliability and life span is a difficult process. How can future users and operators, their needs, and characteristics of their work be analyzed and understood? Although adaptive systems may solve some of such issues, there is a strong need for intelligent and cognitive systems to help alleviate some of the problems.

Specifications: How can the scope and specifications of intelligent systems be defined and described adequately? A large portion $(84 \%)$ of today's designs of simple systems fails due to incomplete or unclear specifications. On the other hand, how many people read and understand complete specifications in a 1000-page or so document? What methodologies and tools must be developed to help in this mater?

Design and Evaluations: How do we design an interactive, intelligent, or cognitive system optimally for current and future users? It is relatively easy to design complex systems that are complicated and hard-to-use. It is very hard to design complex systems that are neither complicated nor hard-to-operate. The very nature of cognitive systems is to help in this matter.

What new tools should we develop? The tools to design small subsystems are formidable. Assembling such subsystems into larger systems requires many solutions to the management of interfaces between the subsystems and coordination of parallel operations between the subsystems. What method and strategies must be introduced to predict and prevent errors in product development? Are the current solutions [ChIs04] satisfactory for such systems? Would tools such as LabVIEW from National Instruments be helpful in teaching students how to model and evaluate designs in embedded systems and mechatronics?

Hardware and Sensors: Should parallel hardware be used? How should parallel software match the parallel hardware and parallel network? Should the hardware be event or time driven? Should it be synchronous or anachronous? Should it be control or data driven? What granularity should be employed? Should it use crisp or fuzzy logic? Should it be structural (e.g., neural network based)? Should it be purely digital or hybrid (digital and analog)?

Sensing of the environment is central to intelligent and cognitive machines. How can we provide new reliable sensors with self-calibration, self-correcting, and resilient to aging? How can we improve machine vision, as well as realtime natural speech recognition and understanding? Since single sensors are not sufficient to provide input to cognitive systems, reliable and robust mesh networking is required, both wired and wireless.

Software: Software requires many advances to be suitable for intelligent and cognitive systems. An example of how far we are from this level of sophistication is the low usability of help files in most application software. However, within the EDP itself, the Unified Modelling Language (UML) (e.g., [Doug99]) and similar approaches have been found very useful to build blueprints, with a common vocabulary for software modelling and reduced differences in software methodologies. The UML uses the best practices of the object-oriented software design (OOD) process [Scha99, $\mathrm{p}$. 82], [Doug99]. The OOD is fundamentally different from the functional design process. It emphasizes objects rather than functions. Objects identify both data (attributes) and methods (functions) that can act on the data. Objects are instances of a class (with the same attributes and methods). An object encapsulates this information, and cannot change it by itself. This encapsulation improves the reliability and 
maintainability of software, as changes appear to be local.

\section{CHALLENGES IN EDUCATING ENGINEERS FOR COMPLEX SYSTEMS}

\subsection{Methodological and Innovative Design}

Our engineering students have an extensive exposure to mathematics (algebra, calculus, analysis, and discrete mathematics), basic sciences (physics, chemistry, and some geological and environmental sciences), and basic engineering sciences (electronics, computing, signal and image processing, information theory, coding, electromagnetics, telecommunications, and computer networking). This exposure in itself does not convert a student into and engineer; it just provides the required scientific and engineering knowledge. This model is well established in many countries [CEAB06], [ABET06], [DWCP02].

Any good engineering program must also incorporate the methodological application of the knowledge in order to design and produce a new device, system, or process, each satisfying human needs and safety. In addition, for a program to be excellent, it should develop the concept of innovative design (which is in some contradiction with the concept of methodological design). This is of particular importance to the design of intelligent systems that must be autonomous, autonomic, survivable, immune to external and internal attacks, and recognize the changing human needs and safety. How do we teach and develop such design skills?

\subsection{Extended Coverage}

The design of intelligent and cognitive systems requires a major extension of the topics taught to our students, including intelligent signal processing [HPSM06], biology, cognitive informatics [Wang03], and new computing paradigms such as structural computing (i.e., neural networks, particularly with recurrence and full connectivity in which emergence can occur), as well as quantum and biomolecular computing. In signal processing, we already teach autocorrelation, convolution, and Fourier transforms, but must extend it to time-scale (wavelet) transforms [Mal198]. Control theory should be extended to include nonlinear dynamical systems with the fourth stable state, chaos. This necessitates extensions to discrete mathematics to include self-affinity, multiscale analysis, fuzzy logic, and higher-order statistics. Electronics must also emphasize the physics of computing and discuss challenges in high-speed circuit design.

\subsection{New Emerging Areas vs Specialization}

How do we respond to the emerging areas of engineering such as Genetic Engineering, Cognitive Engineering? Will biology, neuroscience, bioinformatics, cognitive informatics be all required as Basic Sciences? Will Computer Science be ever considered as one of the Basic Sciences?
How do we distinguish between a new emerging area (discipline) and a highly-specialized sub-area? Many offshore engineers graduate from very narrow specialties. Does this approach (narrow, but deep) help or hinder the design process? Will teams be helped by such narrow specialties? Do broadly-based engineers defined by their discipline adapt to change faster?

\subsection{Experiential Learning}

How do we deal with experiential learning (i.e., co-op terms, 16-month internship, stage projects)? Companies no longer tolerate extended years of on-the-job training for new engineering graduates because of the short product life cycles, fast turn-around of graduates (the old model of seven years with a company no longer applies). The recent shift from a graduating thesis to a capstone project based on teams who practise the engineering design process is a reflection on how engineering schools cope with the problem.

A good example of an experiential learning capstone project is the design, implementation of a variable lift vehicle for near-space applications [ADMK11]. The team of three students from the Department of Electrical \& Computer Engineering, University of Manitoba (UofM) designed and developed the mechanical and electronic parts of the system in time to test it. Since the vehicle was tested in non-near-space conditions, the requirements have changed, and the experiments had to be modified. The resulting experience matched many real industrial environments.

However, the capstone projects last one year only. Could the experiential learning concept be extended to the entire educational program, spanning four years? An example of such a multi-year project is the T-Sat project at the UofM [KBSD11]. The project is to design, implement, and test a triple picosatellite (code T-Sat) under the umbrella of the first Canadian Satellite Design Challenge that started in 2010. There are 13 teams in Canada who have joined the competition. A successful satellite will be launched in two years. The UofM T-Sat team has 79 registered undergraduate and graduate students from various faculties and schools, including engineering, sciences, business, and arts. Most of the students are from senior years, but some are also from the first year. The graduate students are often the leads in various sub-teams. We also have over 49 project advisors, including academic, aerospace, industrial, radio, military, government and community. This multi-team project has the potential of bringing experience to the teams that far exceed the capstone experience. It provides an opportunity to the industries to participate in the experiential learning process directly through their advisors. It provides the students with direct knowledge of the companies through their advisors.

There are also other design competitions intended to increase the experiential learning, including the Society for 
Automotive Engineers (SAE) Solar Car and the FormulaSAE, or the NASA's Space Elevator.

\section{RESEARCH CHALLENGES}

The engineering design process (EDP) is used in industry, research and development (R\&D) institutions, research laboratories, educational institutions, and many other places where new concepts must be developed and made operational. We have seen that the process itself is multi-facet and requires much knowledge and experience. How are the EDP tools and methodologies developed? Is there a room for research related to EDP?

The Cambridge Engineering Design Centre [CEDC07] has been conducting research in several areas. For example, their knowledge management includes acquisition, storage, and retrieval of engineering knowledge related to design. Process management addresses the modelling aspects of the design process. Change management studies how to model change in products. Computational design focuses on integrated optimization methods and tools. Design practice has been developing understanding of the practices used in the design process.

\section{CONCLUDING REMARKS}

Complex machines and systems are no longer designed and built to serve users, but are developed for operators and humans who play an integral part in the systems. This concept goes beyond the traditional human-machine interface whose function is to match the two distinct entities. Since complex machines are inherently emerging, traditional design approaches are inadequate. Intelligent signal processing, real-time machine learning, and cognitive informatics are essential in solving some design and implementation issues of adaptive, intelligent and cognitive machines. The engineering design process (EDP) is critical to the development and introduction of such machines and systems. Since the classical EDP models have not been involved with the design of such complex machines, a new improved paradigm must be developed. This paper addresses some of the challenges of developing the new paradigm.

\section{Acknowledgements}

Partial financial support of this work from the Natural Sciences and Engineering Research Council (NSERC) of Canada is gratefully acknowledged.

\section{References}

[ABET06] ABET Engineering Accreditation Commission, "Criteria for accreditation engineering programs," Report, 2006, 29 pp. Available as of June 2007 from

http://www.abet.org/forms.shtml

[Alek98] Igor Aleksander, "From WISARD to MAGNUS: A family of weightless neural machines," in [Aust98; pp. 1830].

[ADMK11] Kane Anderson, Tahir Diop, Yin Fei Meng, and
Witold Kinsner, "Collaborative learning through variablelift control for near-space applications," in the Proc. Can. Engineering Education Confrence, CEEC11 (St. John's, NL; June 6-8, 2011) 2011.

[Bolt03] William Bolton, Mechatronics:Electronic Control Systems in Mechanical and Electrical Engineering. Edinburgh Gate, UK: Pearson Education, 2003 ( $3^{\text {rd }}$ ed.), 574 pp. \{ISBN0-131-21633-3 pbk\}

[Broo 75] Frederick P. Brooks, The Mythical Man Month. Reading, MA: Addison-Wesley, 1997, 195 pp. \{ISBN 0201-00650-2 pbk\}

[Broo 10] Frederick P. Brooks, The Design of Design: Essays from a Computer Scientist. Reading, MA: Addison-

Wesley, 2010, 448 pp. \{ISBN 978-0-201-36298-5 pbk\}

[CEDC07] Cambridge Engineering Design Centre,

"Research," Report. Cambridge, UK: Cambridge

Engineering Design Centre, 2007. (Available as of June 2007 from

http://www-edc.eng.cam.ac.uk/research/)

[CEAB06] Canadian Engineering Accreditation Board,

"Accreditation criteria and procedures," Report, 2006, 40

pp. Available as of June 2007 from

http://www.engineerscanda.ca/e/prog_publications_3.cfm

[Ceti07] Sabri Cetinkunt, Mechatronics. New York, NY:

Wiley, 2007, 615 pp. \{ISBN 978-0-471-47987-1\}

[ChIs04] Lawrence P. Chao and Kosuke Ishii, "Design process error-proofing: Challenges and methods in quantifying design elements," in Proc. Tenth ISSAT Intern. Conf. on Reliability and Quality in Design (Las Vegas, NV; August 2004) 5 pp., 2004. \{ISBN 096399980903 (Available as of June 2007 from http://www-

mml.standford.edu/publications/2004/ISSATquality-C0424.pdf)

[Coff00] Ken Coffman, Real-World FPGA Design with Verilog. Upper Saddle River, NJ: Prentice Hall, 2000, 289 pp. \{ISBN 0-13-099851-6,+CDROM $\}$

[Cros00] Nigel Cross, Design Methods: Strategies for Product Design. New York, NY: Wiley, 2000 ( $3^{\text {rd }}$ ed.), 224 pp. \{ISBN 0-471-87250-4\}

[DWCP02] Samantha De Bon, Deborah Wolfe, Jean-Yves Chagnon, and William G. Paterson, "Engineering Accreditation in Canada and Its Current Challenges," in Proc 2002 ASEE/SEFI/TUB Colloquium, 9 pp. 2002. (Available as of May 2007 from http://www.asee.org/conferences/international/papers/uplo ad/Engineering-Accreditation-in-Canada-and-Its-CurrentChallenges.pdf)

[Doug99] Bruce Powel Douglass, Doing Hard Time: Developing Real-Time Systems with UML, Objects, Frameworks, and Patterns. Reading, MA: AddisonWesley, 1999, 749 pp. \{ISBN 0-201-49837-5,+CDROM\} [Duec01] Robert K. Dueck, Digital Design with CPLD Applications and VHDL. Stamford, CT: Thomson Learning, 2001, 845 pp. \{ISBN 0-7668-1160-3, 
$+\mathrm{CDROM}\}$

[EdHo08] W. Ernst Eder and Stanislav Hosnedl, Design Engineering: A Manual for Enhanced Creativity. Boca Rayton, FL: CRC, 2008, 588 pp. \{ISBN 978-1-42004765-3 \}

[FoCo05] Ralph M. Ford and Chris S. Coulston, Design for Electrical and Computer Engineers: Theory, Concepts, and Practice. New York, NY: McGraw Hill (Custom Publishing), 2000, 280 pp. \{ISBN 0-07-319599-5 pbk\}

[Fran95] Stan Franklin, Artificial Minds. Cambridge, MA: MIT Press, 1995, 464 pp.

[GaCo03] A.G. Ganek and T.A. Corbi, "The dawning of the autonomic computing era," IBM Systems J., vol. 42, no. 1, pp. 34-42, 2003. (Available as of May 2006 from http://www.research.ibm.com/journal/sj/421/ganek.pdf)

[Haik03] Pentti O.A. Haikonen, The Cognitive Approach to Conscious Machines. New York, NY: Academic, 2003, 294 pp. (See also http://personal.inet.fi/cool/pentti.haikonen/)

[Hayk05] Simon Haykin, "Cognitive radio: Brainempowered wireless communications," IEEE J. Selected Areas in Communications, vol. 23, no. 2, pp. 201-220, February 2005.

[Hayk06] Simon Haykin, “Cognitive radar,” IEEE Signal Processing Mag., pp. 30-40, January 2006.

[HaCh05] Simon Haykin and Zhe Chen, "The cocktail party problem," Neural Computation, vol. 17, pp. 1875-1902, 2005.

[HPSM06] Simon Haykin, José C. Principe, Terrence J. Sejnowski, and John McWhirter, New Directions in Statistical Signal Processing. Cambridge, MA: MIT Press, 2006, 544 pp.

[HoWo05] Eric Hollnager and David D. Woods, Joint Cognitive Systems: Foundation of Cognitive Systems Engineering. Boca Rayton, FL: CRC, 2005, 240 pp. \{ISBN 0-849-32821-7\}

[IBM06] IBM Autonomic Computing Manifesto. 2006 (Available as of May 2006 from http://www.research.ibm.com/autonomic/)

[IBM07] IBM, "Engineering design: Clash analysis in automotive and aerospace," 2007. (Available as of May 2007 from http://www-

03.ibm.com/grid/solutions/ED_clashanalysisautoaero.shtm 1)

[ITEA07] International Technology Education Association, "Glossary terms for STL and AETL and Addenda," Report, 2007, 30 pp. (STL -Standards for Technological Literacy; AETL -Advancing Excellence in Technological Literacy; TAA - Technology for All Americans)

Available as of June 2007 from

$<$ http://www.iteaconnect.org/TAA/Publications/TAA_Pub lications.html>

[Kins05] Witold Kinsner, "Signal processing for autonomic computing," in Proc. 2005 Meet. Can. Applied \& Industrial Math Soc., CAIMS 2005 (Winnipeg, MB; June
16-18, 2005) 2005. Available as of May 2006 from http://www.umanitoba.ca/institutes/iims/caims2005_them e_signal.shtml

[Kins07] Witold Kinsner, "Towards cognitive machines: Multiscale measures and analysis," Intern. J. Cognitive Informatics and Natural Intelligence, vol. 1, no. 1, pp. 2838, 2007.

[Kins09] Witold Kinsner, "Challenges in the design of adaptive, intelligent and cognitive systems," Intern. J. Software Science \& Computational Intelligence, vol. 1, no. 3, pp. 16-35, July-Sept. 2009.

[KBSD11] Witold Kinsner, M.D. (Ron) Britton, Dario Schor, Arash Fazel Darbandi, Kris Goodmanson, Cody Friesen, and Emily Bashford, "Preliminary experience from the T-Sat project," in the Proc. Can. Engineering Education Confrence, CEEC11 (St. John's, NL; June 6-8, 2011) 2011.

[Koen03] Billy Vaughn Koen, Discussion of the Method: Conducting the Engineer's Approach to Problem Solving. Oxford, UK: Oxford University Press, 2003, 260 pp. \{ISBN 0-19-515599-8 pbk\}

[Leon95] Leonhard Center for the Advancement of Engineering Education, "World-class engineer," Report, The Pennsylvania State University, December 1995. Available as of June 2007 from $<$ http://www.engr.psu.edu/AboutCOE/worldclass.asp $>$

[Mal198] Stéphane Mallat, A Wavelet Tour of Signal Processing. San Diego, CA: Academic Press, 1998, 577 pp.

[Scha99] Stephen R. Schach, Classical and Object-Oriented Software Engineering with UML and Java. New York, NY: McGraw Hill, 1999 (4 ${ }^{\text {th }}$ ed.), 616 pp. \{ISBN 0-07230226-7; +CDROM\}

[Tayl03] John G. Taylor, "The CODAM model of attention and consciousness," in Proc. Intern. Joint Conf. Neural Networks, IJCNN03 (Portland, OR; 20-24 June 2003) vol. 1, pp. 292-297, 2003.

[Wang02] Yingxu Wang, "The real-time process algebra (RTPA)," Intern. J. of Annals of Software Engineering, vol. 14, no. 10, pp. 235-274, October 2002.

[Wang03] Yingxu Wang, "Cognitive informatics models of software agent systems and autonomic computing: Keynote Speech", in Proc. Intern. Conf. Agent-Based Technologies and Systems (ATS'03), U of C Press, Calgary, Canada, August, p. 25, 2003.

[Wang04] Yingxu Wang, "On autonomic computing and cognitive processes: Keynote speech", in Proc. 3rd IEEE Intern. Conf. on Cognitive Informatics, ICCI04, (Victoria, BC; 16-17 August 2004) ISBN 0-7695-2190-8, pp. 3-4, 2004.

[Wang06] Yingxu Wang, Cognitive Informatics - Towards the future generation computers that think and feel: Keynote speech", in Proc. 5th IEEE Intern. Conf. Cognitive Informatics, ICCI06, (Beijing, China;, July 1719, 2006) ISBN 1-4244-0475-4, pp. 3-7, 2006. 